\title{
La clôture des religieuses et les rapports de genre dans les couvents italiens (fin XVIe-début XVIIe siècles)
}

\section{Gabriella Zarri}

\section{(2) OpenEdition}

Journals

Édition électronique

URL : https://journals.openedition.org/clio/5492

DOI : 10.4000/clio.5492

ISSN : 1777-5299

Éditeur

Belin

Édition imprimée

Date de publication : 1 novembre 2007

Pagination : 37-60

ISBN : 978-2-85816-940-5

ISSN : 1252-7017

Référence électronique

Gabriella Zarri, «La clôture des religieuses et les rapports de genre dans les couvents italiens (fin XVle-début XVIle siècles) », Clio. Femmes, Genre, Histoire [En ligne], 26 | 2007, mis en ligne le 01 janvier 2010, consulté le 23 avril 2022. URL : http://journals.openedition.org/clio/5492 ; DOI : https://doi.org/ $10.4000 /$ clio.5492

Ce document a été généré automatiquement le 23 avril 2022

Tous droits réservés 


\title{
La clôture des religieuses et les rapports de genre dans les couvents italiens (fin XVIe-début XVIIe siècles)
}

\author{
Gabriella Zarri
}

\section{NOTE DE L'ÉDITEUR}

Traduit de l'italien par Christiane Klapisch-Zuber

1 L'obligation de la clôture des religieuses fut proclamée par le décret « Des réguliers et des moniales » voté lors de la XXVe session du Concile de Trente, qui se tint les 3 et 4 décembre 1563 et fut la dernière assemblée du concile ${ }^{1}$. La réforme des réguliers et des religieuses était une question d'importance au sein de l'Église et ses implications sociales étaient grandes. Au cours des discussions, les évêques avaient émis des opinions discordantes sur la radicalité des dispositions à adopter. Le décret résulta d'un compromis qui porta dans deux directions: la libre acceptation de la profession religieuse, d'une part, et la discipline, de l'autre. Pour arrêter les vocations forcées, on exigea un âge plus élevé à la profession tant des hommes que des femmes; quant à ces dernières, l'évêque ou son délégué devait en outre bien examiner si leur choix d'entrer au couvent était libre. Sur le plan de la discipline, on réduisit ou révoqua la licentia extra standi accordée aux religieux, c'est-à-dire la faculté de vivre hors du couvent pour étudier ou exercer un office auprès d'une cour seigneuriale ou d'une famille privée ${ }^{2}$; l'obligation de la clôture fut restaurée pour les moniales, et la responsabilité de la surveillance de son application fut confiée à l'évêque, à qui échut aussi la responsabilité des monastères soumis à la juridiction d'un ordre religieux. Dans la mesure où il fut le fruit d'un compromis, le décret adopté à Trente contenait de sévères dispositions à l'égard des pratiques contemporaines, dispositions qui furent par la suite confirmées et 
précisées en ce qui concerne la clôture des religieuses, et aggravées par une série de bulles papales et de décisions de la Sacrée Congrégation du Concile³.

Les historiens ont porté des appréciations variées sur l'efficacité du décret tridentin concernant les moines réguliers et la clôture des moniales en Italie. Depuis les années 1990, la clôture des religieuses après le Concile de Trente a constitué l'un des problèmes les plus discutés des women's studies. Reprenant aussi les thèses de Michel Foucault, ces travaux ont surtout relevé l'analogie avec l'»enfermement» des populations marginales propre à l'époque moderne. Ce furent ainsi le contrôle et la répression que l'on mit essentiellement en lumière dans la clôture, en recherchant les témoignages qui désignaient le monastère comme une prison ou un "enfer ", dans le sillage de sœur Arcangela Tarabotti, cette nonne du XVIIe siècle vénitien qui donna deux visions opposées du cloître - paradis ou enfer monacal ${ }^{4}$.

Dans la veine qu'on peut dire "répressive" de ces interprétations, l'enquête a également porté sur les cas de rébellion contre la clôture ${ }^{5}$ ou sur les demandes de sortie du couvent adressées à la Sacra Penitenziaria par des religieuses des XVe et XVIe siècles ${ }^{6}$. Le nombre restreint de demandes de dispense des vœux monastiques retrouvées par les recherches les plus récentes déplace cependant le cœur du problème historique de la rébellion contre la clôture ${ }^{7}$. Il s'agit moins, désormais, de cerner des individualités singulières et décrire la révolte de moniales contre leur destin personnel que d'analyser la structure sociale globale de l'Ancien Régime, le pacte, en particulier, que l'État et l'Église, dans les pays catholiques, scellèrent en vue de préserver l'ordre des institutions ecclésiastiques et de garantir et consolider la famille patrilinéaire - et cet ordre impliquait le système des bénéfices du clergé aussi bien que les monastères et congrégations de femmes ${ }^{8}$. De ce point de vue, la clôture monastique imposée par le Concile de Trente apparaît comme une mesure de caractère disciplinaire dont l'application a directement intéressé les autorités ecclésiastiques et les magistratures citadines, qui s'accordaient pour ne pas laisser d'alternative aux individus. Toutefois, si l'observation de la clôture s'est trouvée réglée formellement par les lois ecclésiastiques, elle s'est caractérisée par la tolérance de concessions, dérogations et privilèges adoucissant les limites entre la pratique et la norme.

4 Sur la base d'un plus grand nombre de cas et d'une littérature aujourd'hui plus abondante, l'historiographie actuelle ne considère plus la clôture comme une barrière insurmontable qui aurait totalement coupé les liens avec la famille et la société ; elle adopte plutôt le concept de "perméabilité" pour désigner un état de relative communication entre le dedans et le dehors du monastère ${ }^{9}$. L'histoire des femmes en est arrivée à cette idée de perméabilité à partir du concept plus général de "négociation", dans lequel les études féministes récentes voient un caractère intrinsèque du pouvoir des femmes tel qu'il s'exprime dans la sphère privée et familiale ${ }^{10}$. Dans la mesure où les institutions ecclésiastiques sont impliquées, la négociation ne joue toutefois ici que de façon limitée et secondaire face à un appareil législatif et institutionnel qui, pour toute requête et décision, engage une action exclusivement gérée par des hommes, du confesseur à l'évêque et aux cardinaux des Sacrées Congrégations romaines. Mais est-il réaliste de recourir à la notion de négociation à propos d'un bouleversement aussi radical que fut la séparation du monde décrétée par le Concile de Trente?

5 Parmi les nouvelles interprétations qui ont été avancées sur les monastères féminins de l'époque tridentine, on signalera celle d'un «troisième espace» pour les femmes, 
alternative à la famille et à la profession monastique, formulée par Camilla Russell dans ses travaux sur Giulia Gonzaga, une jeune veuve dévote qui vers la moitié du XVIe siècle choisit pour cadre de vie et de ses rencontres spirituelles un monastère de tertiaires ${ }^{11}$. Mais ce sont surtout les études sur l'architecture monastique, interprétée par Helen Hills à la lumière de l'histoire du gender, qui retiennent l'attention. L'examen attentif des monastères napolitains du XVIIe siècle, caractérisés par leurs très hauts portails, a permis à la chercheuse anglaise d'interpréter le monastère comme le corps féminin de la ville, architecturalement présenté comme un corps virginal ${ }^{12}$. Enfin, aux enquêtes sur les monastères de femmes et sur la clôture qui s'inspirent des méthodes et des questions de l'histoire sociale ${ }^{13}$ ou de l'histoire des femmes ${ }^{14}$, je me permettrai d'ajouter l'étude que j'ai menée il y a quelques années dans une perspective d'histoire du gender et sur laquelle je reviendrai plus loin ${ }^{15}$.

6 Ces remarques m'engagent à approfondir ici les changements que la clôture tridentine a introduits dans les rapports de genre, tant dans les relations avec le monde extérieur que dans le microcosme monastique. Du point de vue documentaire, je concentrerai mon enquête sur deux villes italiennes, Florence et Bologne, dont les archevêques se distinguèrent, entre le dernier quart du XVIe siècle et les premières décennies du XVIIe, par l'attention qu'ils portèrent à la mise en œuvre des décrets conciliaires et par le souci qu'ils manifestèrent personnellement dans leur correspondance à l'égard du gouvernement des religieuses.

La clôture et le monde extérieur

7 Quand il établit les normes de la construction d'un monastère bien réglé, Charles Borromée (1538-1584) indiqua comme première et plus importante condition le « site " où il serait installé : loin des édifices religieux masculins, des tours et murs de la ville, d'édifices plus élevés d'où les moniales pourraient être vues; séparé des places, marchés et lieux fréquentés ou bruyants ; situé néanmoins dans une position évidente et non pas dissimulée. Un site qui permettrait en somme l'isolement et le recueillement sans être séparé de la ville où les monastères féminins devaient être une présence visible. Dans ses Instructions pour les constructions religieuses ${ }^{16}$, modèle que la curie romaine devait adopter, on ne trouve pas mention des murs qui devaient entourer le monastère ; ils étaient, en revanche, l'élément essentiel et déterminant qui retenait l'intérêt des visiteurs apostoliques et des évêques locaux appelés à vérifier l'application des normes tridentines dans les édifices conventuels. Sans aucun doute, les murs des monastères constituaient depuis le bas Moyen Âge une structure identifiant clairement les lieux de religion, surtout féminins. De fait, dans la peinture du XVe siècle, le « jardin clos » traduisait visuellement l'image biblique du hortus conclusus, interprété sur le plan spirituel comme Paradis et sur le plan anthropologique comme jardin virginal; une image qui renvoyait en premier lieu aux monastères, dans les pays méditerranéens, et aux béguinages dans les pays d'Europe du Nord, d'où nous sont parvenus les témoignages les plus extraordinaires de ces peintures. Pourtant, dans la période posttridentine, la représentation du monastère comme hortus conclusus recule, sans disparaître complètement, devant celle de la citadelle assiégée, dont la muraille prend toujours plus valeur de protection et séparation.

8 Si les normes architecturales borroméennes ne mentionnent pas les murs des monastères, leurs dispositions se font minutieuses en ce qui concerne les portes, les fenêtres du parloir et les "tours $»^{17}$, principaux points de contact entre l'intérieur et l'extérieur. Près de la porte, une petite cellule était prévue pour la sœur tourière, et en 
face était situé le « tour » dont la taille et la forme étaient décrites de façon détaillée. La porte et le tour du monastère devinrent des endroits marqués par une surveillance continue, qui, cela dit, s'exerçait par de rares ouvertures. Les occasions d'ouvrir la porte du monastère étaient en effet peu nombreuses. Sauf dans les cas exceptionnels, tels qu'incendies, épidémies ou pestes, les portes ne devaient s'ouvrir devant les moniales que lors de leur entrée au couvent et à leur mort, lorsque leur dépouille était portée à l'église extérieure. Les seules personnes autorisées à franchir le seuil étaient l'évêque et son vicaire pour les visites pastorales, le confesseur venu porter l'extrême onction aux mourantes ou confesser les infirmes, et le médecin pour soigner les malades. Les ouvriers, jardiniers ou paysans pouvaient entrer si nécessaire par la porte dite « des charrettes ", par où ils accédaient aux granges, potagers et jardins.

9 Dans le quotidien du monastère, diverses personnes pouvaient avoir besoin de s'entretenir avec les nonnes, depuis les administrateurs séculiers des biens monastiques ou des propriétés rurales, jusqu'aux vendeurs des denrées les plus variées, ou aux parents des professes. Toutes ces personnes pouvaient rencontrer les religieuses dans une "cella collocutionis", autrement dit dans un parloir qui devait avoir un accès direct sur l'extérieur et des fenêtres s'ouvrant sur l'intérieur, munies de grilles très serrées recouvertes de toile noire.

Cette nécessité de surveiller les portes, les tours et les parloirs conduisit à établir de nouvelles charges au sein du monastère et contribua aussi à altérer la hiérarchie traditionnelle des offices. Si la possession des clefs attribuée à l'abbesse lors de son élection signifiait essentiellement qu'elle était investie du gouvernement du monastère, le contrôle de la clôture conférait aux sœurs tourières et aux auditrices ${ }^{18}$ une importante coresponsabilité dans le maintien de la discipline ecclésiastique.

11 Parmi toutes les personnes ayant accès au monastère, le confesseur jouait un rôle particulier. Il disposait d'un logement adjacent au monastère, sans communication directe, cependant, avec lui. Il était en principe âgé, mais devait être en mesure de confesser un nombre parfois élevé de religieuses et de leur donner la communion. Il allait prendre une importance croissante dans les monastères de femmes de la ContreRéforme. Sa tâche principale était de maintenir l'harmonie et la sérénité dans le couvent où il ne devait pas fomenter de factions, ni alimenter les scrupules ou les enthousiasmes religieux excessifs, mais diriger avec discernement les moniales que parfois affectaient des maladies «de l'esprit » comme la mélancolie ou la possession diabolique.

Bien que la réforme tridentine eût prescrit la mise en conformité de tous les monastères avec des normes architecturales garantissant l'inviolabilité du lieu consacré, les accès au monastère et au parloir n'étaient pas libres, et l'autorité ecclésiastique les contrôlait dans le menu détail. Toute personne qui avait à parler avec les moniales ou à entrer dans le monastère en raison de ses fonctions devait être munie d'un laissez-passer en règle délivré par la chancellerie épiscopale. Les registres de la Curie archiépiscopale de Ferrare où étaient notés les noms des personnes autorisées à entrer dans un couvent révèlent un va-et-vient fréquent, voire quotidien, de médecins, paysans, ouvriers, maçons, peintres et organistes, qui venaient, parfois des mois durant, exécuter des travaux à l'intérieur de l'enceinte et des quartiers conventuels ${ }^{19}$.

13 Il ne fut pas davantage interdit aux religieuses de garder des fillettes pour leur éducation ni d'héberger provisoirement, parfois même durablement, de nobles dames, veuves ou mariées. Ce qui changea radicalement ce fut l'autonomie des moniales, 
constamment obligées à demander à leurs supérieurs, l'évêque ou même le Pape, et à la Congrégation romaine des Évêques et Réguliers l'autorisation d'exercer ces services, traditionnels pour elles, d'hospitalité et d'assistance aux femmes en difficulté. Les témoignages sont à cet égard innombrables.

Aux Murate de Florence ${ }^{20}$, seules les négociations autour de la dot d'une novice étaient désormais de la compétence de l'abbesse et tout autre engagement était nul et non avenu s'il allait à l'encontre d'une observation intégrale de la règle ou s'il n'était pas autorisé comme le leur rappelait fermement en octobre 1593 l'archevêque de Florence Alexandre Médicis (1535-1605), futur pape Léon $\mathrm{XI}^{21}$. Des femmes pouvaient certes être admises dans le monastère, mais si elles voulaient y demeurer durablement, elles devaient prononcer leurs vœux et se soumettre à la clôture monastique ${ }^{22}$.

côture tridentine n'élimina donc pas la possibilité de loger des femmes du siècle dans un monastère mais, déléguant aux supérieurs ecclésiastiques la décision concernant leur entrée et la durée de leur séjour, elle filtra soigneusement identités et motivations. Un dépouillement attentif des réponses des Sacrées Congrégations du Concile et des Evêques et Réguliers aux questions posées entre 1573 et 1635 par les différents archevêques de Bologne sur la clôture des nonnes révèle qu'une bonne partie des demandes en provenance de cette curie concernait la permission de loger dans le monastère des veuves solitaires ou accompagnées d'une servante, des femmes maltraitées par leur époux et en instance de séparation, ou encore de nobles dames dont le mari était absent pour des raisons diverses.

Tous ces témoignages confirment l'interprétation avancée sur la «perméabilité » du monastère même après le Concile de Trente ; ils étayent l'hypothèse d'un " troisième espace » offrant aux femmes une alternative au mariage ou à la profession monastique, même si l'on constate en parallèle que la clôture eut pour effet de réduire nettement les capacités des femmes à négocier et d'installer une domination masculine plus marquée sur leurs établissements.

La clôture au dedans

17 Dans une lettre expédiée de Rome le 3 mars 1576, l'archevêque de Florence, Alexandre Médicis, rappelle à l'abbesse des Murate la haute responsabilité attachée à sa fonction et l'exhorte à être "une mère et une sœur, non pas une reine " ${ }^{23}$. Étant donné que la figure exprimant la «forme de vie» des établissements monastiques était la République, l'exhortation à l'abbesse à ne pas agir en "reine» mettait en lumière l'éternel danger de faire de l'exercice du pouvoir une tyrannie. Parmi toutes les sources qui attestent la diffusion de l'image du monastère comme République, je mentionnerai la définition que le même Alexandre Médicis formulait dans son Traité sur le gouvernement des Monastères composé en 1601 et adressé à son Vicaire. Pour lui, la vie monastique était doublement connotée: comme "vie angélique », qui renvoyait au concept déjà mentionné du "jardin clos» ou Paradis, et comme "République », qui faisait au contraire référence à un gouvernement du monastère caractérisé par la vie commune et la collégialitée ${ }^{24}$.

18 On sait que les décrets du Concile de Trente imposèrent non seulement la réactualisation de la clôture, mais l'observation des vœux monastiques. Toutefois, la mise en œuvre de la vie commune liée au vœu de pauvreté rencontra des obstacles qui se traduisirent au début par des difficultés économiques sévères dans les monastères de femmes, et plus encore par la persistance de leurs traditions propres. L'origine noble de la plupart des professes conduisit les autorités ecclésiastiques à une sorte de 
compromis sur ce point et à une relative tolérance envers la possession de biens matériels25. En revanche, les normes réglant l'âge de l'entrée au monastère et l'âge minimal de la prise de voile et de la profession eurent plus de succès. Après le concile, les couvents ne purent plus accueillir de fillettes de moins de sept ans ou de femmes séculières de plus de vingt-cinq ans à des fins d'éducation. De plus, la profession dut être faite à un âge qu'on pourrait appeler « du consentement ", c'est-à-dire de celui où un jeune pouvait répondre de ses choix et dénoncer éventuellement les violations de sa liberté, âge que le concile situa à seize ans pour les deux sexes. Élèves et novices durent en outre habiter dans des parties déterminées du monastère, séparées des professes ${ }^{25}$ : cette règle devait empêcher que se constituent des groupes familiaux compacts; elle devait aussi encourager l'acquisition d'une formation culturelle et religieuse commune, répondant aux modernes critères pédagogiques introduits par l'humanisme et traduits dans la pratique de la ratio studiorum des Jésuites.

Dans l'espace clos du monastère d'après Trente, on redistribua aussi l'espace intérieur pour donner plus d'importance aux lieux destinés à des activités communes, comme la salle capitulaire, le réfectoire ou la salle de travail, ou pour créer ex novo des quartiers réservés aux jeunes. À la disposition hiérarchisée traditionnelle fondée sur une double division, horizontale entre professes et converses, et verticale en fonction de l'entrée en religion, était désormais juxtaposé un troisième agencement défini par la date de naissance. Changement majeur, appelé à influer sur les relations internes de la communauté. Dans les plus anciennes règles monastiques, aussi bien masculines que féminines, l'âge ne constituait pas un élément déterminant, ni pour l'entrée dans la communauté, ni pour l'élection aux charges de plus forte responsabilité. On observera avec intérêt que la règle bénédictine pour les femmes ne prévoyait pas de normes spécifiques quant à l'âge de l'abbess ${ }^{26}$. Dans l'économie spirituelle du monastère, dont le gouvernement était inspiré par Dieu, l'abbesse devait surtout être dotée de discernement, une vertu indépendante de l'âge, alors que la prieure et la tourière étaient élues parmi les sœurs professes âgées : la première devait en effet avoir de l'expérience, et la seconde être dépourvue d'attraits, car c'était elle qu'on chargeait des rapports du monastère avec le monde extérieur ${ }^{27}$.

L'introduction dans le monastère d'un critère comme celui de l'âge, différent de ceux qui traditionnellement fondaient l'organisation de la communauté, entraîna la formation de nouveaux types de groupement. La prévalence des liens familiaux que les dispositions de Trente avaient cherché à limiter en n'accordant qu'à deux sœurs biologiques de faire profession par monastère, fit peu à peu place à une solidarité par classes d'âge et à la compétition entre jeunes et vieilles. C'est ce qu'exprime fort clairement Cecilia Banci, une religieuse de Santa Cristina, monastère camaldule de Bologne, quand l'évêque l'interrogea en 1622 lors d'un grave conflit affectant sa communauté : «L'obéissance est ignorée et il est couramment dit qu'on n'est plus au temps de saint Benoît, et la jeunesse est élevée en sorte qu'elle devient un peu trop présomptueuse $»^{28}$. Sœur Cecilia regrettait le temps où les élèves étaient instruites par des professes qui étaient presque toujours leurs parentes: "Quant à moi, j'aimerais bien qu'on en tienne comme jadis, c'est-à-dire que chacune [des professes] puisse garder une nièce ou une fille qui l'aimerait bien $»^{29}$. Un mémoire envoyé le $1^{\text {er }}$ décembre 1623 à l'archevêque de Bologne Ludovico Ludovisi (1595-1632) révèle la cristallisation des deux groupes de religieuses, les vieilles et les jeunes. Dans cette lettre signée par les "sœurs âgées de San Ludovico", une religieuse de 68 ans dénonce l'attribution au monastère par l'évêque suffragant d'un confesseur trop jeune, sans expérience, qui 
plaisante trop avec les nonnes; elle suggère au prélat de s'informer et d'ordonner au Vicaire « de soumettre à examen les sœurs âgées zélées, et de mettre à jour le mal, car beaucoup de jeunes sœurs se sont amourachées de [ce confesseur]». Elle conclut en invitant l'archevêque à en nommer un qui ait «plus de cinquante ans face à tant de jeunes nonnes $»^{30}$.

21 La division des couvents entre professes jeunes et vieilles s'enracinait profondément dans les questions de discipline, mais elle s'étendait aussi aux rapports de pouvoir, comme en témoignent les problèmes dénoncés par des professes du même monastère de San Ludovico lors de l'élection d'une abbesse contestée en $1624^{31}$. Les groupes d'âges semblent avoir été la nouvelle forme que prirent les clans à l'intérieur des couvents après le concile. Tandis que s'estompaient les raisons politiques liées aux luttes citadines qui caractérisaient les factions antérieures ${ }^{32}$, les divisions internes surgissaient désormais des questions de discipline. À Santa Cristina, ce fut la musique qui déchaîna dans le couvent une rixe à la suite de laquelle l'Ordre des Camaldules fut privé de sa juridiction sur les nonnes ${ }^{33}$; à San Ludovico, les clans étaient davantage alimentés par une tolérance plutôt large envers l'observation de la clôture et les amitiés des moniales avec des gens vivant dans le siècle.

La réorganisation des espaces intérieurs du monastère post-tridentin concerna également la taille et la disposition des cellules ainsi que la création de lieux de sociabilité communautaire. Les cellules familiales, que des personnes privées subventionnaient, continuèrent à exister ou à être construites, mais plusieurs membres de la famille ne purent désormais y habiter ensemble. De fait, l'une des dispositions les plus sévères en matière de discipline interdit aux professes de dormir à deux par cellule. Il n'est pas de visite pastorale ou d'enquête sur les religieuses qui ne prévoie explicitement de s'informer sur le nombre de lits ou sur les nonnes dormant à deux dans le même lit. La familiarité excessive entre membres d'une communauté de même sexe offrait toujours matière au doute quant à la préservation de la chasteté et elle était occasion de scandale dans un couvent. Une lettre envoyée le 7 mai 1625 par le cardinal Ludovisi à son Vicaire montre que quelqu'un avait dénoncé des abus sur les jeunes élèves ${ }^{34}$. L'attention portée par les autorités ecclésiastiques aux dangers des " amitiés particulières » est également soulignée dans le Miroir religieux des religieuses composé par Giovan Pietro Barco, vicaire de Milan sous l'épiscopat de Federico Borromeo (1564-1631) et imprimé en 1609. Dans la partie consacrée au vœu de chasteté, Barco écrivait :

Celles qui entretiennent une amitié avec des consoeurs particulières et éprouvent à leur égard une affection désordonnée qui les amène à prendre leur parti et à se battre pour elles, pèchent mortellement la plupart du temps, car leur compagnie devient mauvais exemple et objet de scandale. Il peut arriver qu'à l'instigation du Diable elles s'aiment sans retenue et que publiquement 'elles s'embrassent, s'étreignent, se caressent les mains' et se promènent dans le monastère en se tenant la main; auquel cas, outre le péché mortel qu'elles commettraient contre la chasteté, elles introduiraient aussi le péché de scandale, dont il leur faudra particulièrement s'accuser, et leurs confesseurs auront à les interroger sur cela $a^{35}$.

La sexualité au couvent donnait aux évêques aussi matière à enquêter lors de leurs visites pastorales, mais peu de religieuses étaient disposées à en parler. Même cette sœur Cecilia, du monastère bolonais de S. Cristina, qui n'avait pas hésité à affirmer que les jeunes nonnes se montraient désobéissantes et présomptueuses, déclarait à l'évêque que, sur le chapitre de la chasteté, « elle croyait que toutes étaient aussi saintes que le veut le respect de ce lieu $»^{36}$. L'honneur du monastère exigeait que les défaillances les 
plus graves fussent cachées, voire ignorées. Les amitiés particulières n'étaient presque jamais dénoncées, alors même que transparaissent çà et là les frasques de quelques nonnes avec les confesseurs, causes de haines et de désordres au sein des monastères. C'est encore les nonnes bolonaises de San Ludovico qui informaient en 1627 le cardinal Ludovisi du mauvais comportement de leur confesseur: "On m'apprend que le confesseur des nonnes de San Lodovico a mis une diligence extraordinaire à hâter le succès de l'élection de la présente abbesse, et qu'il poursuit souvent de ses assiduités plusieurs jeunes moniales $\aleph^{37}$. Dans les monastères post-tridentins, les histoires de rapports amoureux noués avec des personnes séculières ne manquaient pas, comme cette "si périlleuse affaire " pour laquelle la sœur bolonaise Leonora Rovegli fut privée de l'accès au parloir et placée sous surveillance39. Mais, plus souvent, c'était les confesseurs eux-mêmes qui pouvaient aisément faire passer de la confiance à la passion amoureuse.

En raison de la plus grande sévérité des normes régissant la clôture, la réorganisation de la vie monastique dut créer de nouvelles occasions de vie sociale et prévoir des moments de la journée consacrés à la récréation, généralement dans les cloîtres et les jardins. De fait, des règles de simple hygiène conseillaient que ces femmes qui se côtoyaient nombreuses dans le même édifice sans accomplir d'effort physique passent du moins une partie du jour à l'extérieur. Le cardinal florentin Alexandre Médicis suggérait de créer des parcours de dévotion dans les jardins, d'édifier des «tabernacles où seraient représentés le sépulcre du Christ, la sainte Vierge de Lorette, saint Jacques de Compostelle etc., qui seraient autant de buts de procession les jours de fête "; à l'intérieur de la clôture, on pourrait construire un oratoire où « seraient représentés la crèche du Seigneur avec des figurines vêtues d'étoffe naturelle, qu'on changerait au fil des saisons, ou divers mystères de Jésus Christ $»^{38}$. Les récréations spirituelles comprenaient également des comédies, une spécialité des religieuses de Florence que le cardinal ne désapprouvait pas à condition qu'elles soient données aux temps liturgiques convenables, en particulier durant le carnaval ${ }^{39}$. Pendant les fêtes, la musique et le chant étaient autorisés. La lecture de poèmes, romans ou «livres dogmatiques » était au contraire exclue des divertissements agréés. Conformément aux dispositions de la Congrégation de l'Index, les nonnes ne devaient pas lire la Bible en traduction ${ }^{40}$, et les manuels de confession leur étaient déconseillés, car, à en croire le cardinal, « elles y apprennent des choses qu'il n'est pas bien qu'elles sachent $»^{41}$.

Clôture et identité de genre

Dans une lettre d'encouragement écrite vers 1510 à la sœur ferraraise Laura Boiardi une cousine du poète -, un obscur franciscain, fra Giovanni Francesco de Sarzana, s'efforce de souligner la difficulté de la vie de religion, qu'elle soit le fait d'hommes ou de femmes. Il dégage deux des grandes différences entre la condition des frères et des moniales : le changement continu de couvent et de dépendance pour les premiers, et la clôture pour les secondes. Le franciscain trouve cependant la clôture moins difficile car il pense, en accord avec une construction culturelle de longue durée, que la condition des femmes est d'» être enfermées », quel que soit leur état ${ }^{42}$.

Si l'on veut dégager les racines de l'identité monacale et de la différenciation de genre qu'elle comporte, il faut en effet rappeler l'origine sémantique du terme " moine », mot qui dérive du grec monachos, autrement dit "solitaire»; il faut aussi analyser les diverses formes de vie religieuse qui se sont élaborées au fil du temps. Les rites de la profession monastique me semblent à cet égard fondamentaux, parce qu'ils permettent 
d'identifier des références symboliques différentes pour le moine et pour la religieuse. De fait, la profession des moines, au Moyen Âge, rappelait la symbolique de l'investiture chevaleresque et s'exprimait dans l'obéissance vassalique à l'abbé, mettant l'accent sur le caractère de miles christianus, de soldat chrétien chez le religieux. La profession des moniales, en revanche, se fondait sur les plus anciens rites de consécration des vierges et, désignant la nonne comme sponsa Christi, épouse du Christ, était directement empruntée aux cérémonies du mariage ${ }^{43}$. Tout en étant séparés de la société, et vivant précisément en "solitaires", les moines et les religieux, y compris les frères mendiants, continuèrent depuis le Moyen Âge à jouer un rôle actif dans l'Église et la société, si bien que le concept de séparation du monde s'identifia à la référence directe à un couvent et à l'obéissance à un supérieur. Pour les moniales, au contraire, il se confondit vite avec la clôture. Depuis le VIIe siècle, la règle de Donat en prévoyait l'obligation ${ }^{44}$. Une vie retirée était toutefois exigée des femmes de toute condition ou état, comme le rappelle notre franciscain, et certaines d'entre elles qui désiraient se vouer à Dieu choisissaient parfois une clôture volontaire ${ }^{45}$. Quoi qu'il en fût, les plus anciens rituels de la prise du voile et de la profession des moniales identifiaient le renoncement au monde non pas avec la clôture mais plutôt avec la pauvreté et l'égalité que recélait la symbolique de la prise de voile. Ce n'est qu'avec les rituels accompagnant l'entrée des novices au monastère, rituels qui varient selon l'époque et le lieu, qu'on constate le durcissement des normes tridentines sur la clôture et que le détachement définitif des futures nonnes de leur famille et de leur ville est bien souligné.

L'Ordre à suivre pour l'admission des novices à la profession rédigé par l'archevêque de Florence Alexandre Marzi Médicis (1605-1630) prévoyait une cérémonie tenant très précisément compte des contraintes introduites par la clôture tridentine ${ }^{46}$. Puisque le rite de la consécration des vierges se calquait sur celui du mariage séculier, les pratiques qui l'accompagnaient avaient beaucoup en commun avec la cérémonie des noces ${ }^{47}$. Le rite traditionnel de la traditio sponsae, c'est-à-dire de la remise de l'épouse à son mari, était reproduit lors d'une entrée au couvent, mais il se déroulait non pas dans la maison paternelle mais entre le monastère où la novice avait déjà passé une année probatoire et la porte de l'église extérieure où la liturgie était célébrée; le cortège nuptial était composé non pas par les parents, mais par le confesseur, figure paternelle, par deux chantres et quelques prêtres. Dans cette compagnie solennelle, la novice entrait dans l'église où l'accueillait le prêtre qui commençait alors la messe chantée. À un moment donné, il interrogeait la jeune fille sur sa volonté de prendre l'habit religieux. Après quoi, il lui coupait une mèche de cheveux et procédait à la vêture. À la fin du rituel, le prêtre changeait le nom de la novice et lui attribuait ainsi sa nouvelle identité de religion. Une fois la messe célébrée, le même prêtre raccompagnait solennellement la novice jusqu'à la porte du monastère. C'est là que se déroulait un dialogue rituel, sans correspondance dans la tradition antérieure, qui soulignait, en accord avec les décrets de Trente, la perpétuité de la clôture et la liberté du choix de la vie religieuse. Arrivée à la porte close du monastère, en effet, la novice la frappait en chantant: "Ouvrez-moi les portes de la justice et une fois entrée je confesserai le Seigneur ». De l'intérieur, les moniales lui répondaient: "Cette porte est celle du Seigneur. Les justes la franchiront» et elles ouvraient toute grande l'entrée de la clôture. La novice pénétrait dans le couvent et, s'adressant maintenant au prêtre, elle récitait solennellement les paroles qui la retranchaient à jamais du monde : «Voici le 
lieu de mon repos pour les siècles des siècles. C'est ici que je résiderai puisque je l'ai choisi ». par les décrets tridentins, se trouva toutefois compensée dans les monastères des XVIe et XVIIe siècles par l'accroissement des activités individuelles ou collectives de dévotion et par l'acquisition de compétences telles que la musique ou le chant, qui firent des monastères autant de pôles d'excellence lors des fêtes patronales et liturgiques ${ }^{50}$. Toutes les moniales, toutefois, ne furent pas en mesure de s'adapter aux rythmes serrés et répétitifs des prières en commun, du travail, des entreprises collectives. Certaines s'enfermèrent dans leurs cellules en refusant de se plier à la vie commune, d'autres se vouèrent à l'exercice de la lecture et à l'ascèse en prenant comme modèle une sainte dont elles avaient entendu célébrer les actes, d'autres enfin se retirèrent totalement de la communauté, perdant l'esprit ("fuor di cervello») ou se comportant comme des possédées ${ }^{51}$. À Florence, en 1567, la mort de Sibilla della Rena «fut une libération pour elle et pour son monastère ", San Pietro Martire. Mais le narrateur de la fin de sa vie nota que, "revenant un peu à elle », elle avait " demandé, avec toute sa conscience, et obtenu de recevoir l'extrême-onction et la recommandation de son âme $»^{52}$. Cette conclusion inattendue fait douter de la folie de sœur Sibilla. Il est clair que, même si elle était une rebelle, son cas s'inscrivait dans la symbolique de l'imitation des saints.

31 Les affaires de nonnes possédées ("spiritate») se situent à l'opposé. À en juger par la prudence avec laquelle les évêques et cardinaux de la Sacrée Congrégation suggérèrent, depuis le début du XVIIe siècle, de traiter les professes qu'on croyait tourmentées par les démons, et par leur refus de les exorciser, on serait porté à croire que les manifestations de possession diabolique relevaient de comportements courants dans les monastères. La confirmation nous vient du Traité du cardinal Alexandre Médicis : il ne se limitait pas à déclarer que les "possédées » étaient souvent atteintes d'épilepsie ou de «mal d'utérus », c'est-à-dire d'hystérie, comme le voulait la médecine du temps, il ajoutait que «les femmes recluses désirent le changement, qu'elles voudraient être entretenues de toutes sortes de choses et remarquées pour leur singularité $»^{53}$. Le 
cardinal Ludovisi partageait cet avis quand il interdisait à son Vicaire d'exorciser une moniale «spiritata " " parce qu'il arrive souvent que les diverses affections de l'utérus dont souffrent les religieuses les font paraître possédées, ce qu'elles ne sont pas réellement $"^{54}$. Le cardinal Desiderio Scaglia (1567-1639), un membre influent de la Sacrée Congrégation des Réguliers, s'exprimait dans les mêmes termes ${ }^{55}$. Les cas de possession diabolique collective, qui marquèrent les couvents de France et d'Italie dans la seconde décennie du XVIIe siècle, renforçaient la conviction que les religieuses voulaient être "remarquées pour leur singularité » et que les affaires de possession cachaient de secrètes et graves raisons de politique ou de juridiction ${ }^{56}$.

La clôture tridentine des moniales ne fut donc pas seulement une mesure de protection que la culture du temps considérait comme convenant aux femmes de toutes conditions, ainsi que l'atteste la lettre du franciscain de Sarzana à sœur Laura Boiardi ; elle eut des aspects disciplinaires rigoureux que les professes ne parvinrent pas toutes à supporter et qui les condamnèrent parfois à la maladie ou à l'isolement de leur entourage ; elle produisit simultanément, à l'intérieur du monastère, des changements culturels permettant à la plus grande partie des moniales non seulement d'acquérir une identité religieuse approuvée par la société, mais de jouer un rôle actif dans leur communauté. La clôture accentua la différence entre la condition du religieux et celle de la nonne. La limitation des autorisations de demeurer hors des couvents ne réduisit pas la grande liberté de mouvement dont les frères continuèrent à jouir. En témoigne un rapport envoyé en 1625 à la Sacrée Congrégation des Réguliers, qui dénonce la conduite scandaleuse d'un carmélite de Bologne, le noble frère Antonio Maria Mezzavacca. Celui-ci entretenait chaque semaine des rapports avec une prostituée et une amitié suspecte avec sœur Fiorella de Reggio Emilia : il avait introduit trois femmes dans la clôture et loué une maison pour y faire bombance et jouer aux cartes; il se promenait masqué au carnaval avec sa prostituée ou d'autres femmes, suscitant «le scandale public et privé »; il se prévalait en outre de ses prérogatives spirituelles pour plier à ses désirs ses pénitentes en leur promettant de les absoudre ${ }^{57}$.

Les mouvements de frère Mezzavacca étaient spatialement plus limités que les continuels changements de couvent dénoncés par le franciscain de Sarzana, ils suffisent pourtant à nous faire comprendre que la clôture monastique devait aussi protéger les moniales de leurs confrères masculins.

\section{BIBLIOGRAPHIE}

AEM : Acta Ecclesiae Mediolanensis ab eius initiis usque ad nostram aetatem, 1892, opera et studio Achillis Ratti, vol. II, Mediolani, ex Typographia Pontificia Sancti Iosephi, col. 1485-1502. 
Aracangeli Letizia, 1996, « Le ragioni politiche della disciplina monastica », in Gabriella Zarri (dir.), Donna disciplina creanza cristiana dal XV al XVII secolo. Studi e testi, Roma, Edizioni di Storia e Letteratura.

BARCO Giovanni Pietro, 1609, Specchio religioso per le monache posto in luce d'ordine dell'illustr. \& reuerendiss. sign. il sig. cardinale Federico Borromeo arciuescouo di Milano, Torino, per Luigi Pizzamiglio, Stampatore Archiepiscopale.

BIONDI Albano, 1991, «L“'inordinata” devozione nella Prattica del Cardinale Scaglia (ca. 1635) », in Gabriella Zarri (dir.), Finzione e santità tra medioevo ed età moderna, Torino, Rosenberg \& Sellier, p. 306-325.

COeD : Conciliorum Oecumenicorum Decreta, 1973, curantibus Josepho Alberigo, Josepho A. Dossetti, Perikle P. Joannou, Claudio Leonardi, Paulo Prodi, consultante Huberto Jedin, Editio tertia, Bologne, Istituto per le Scienze Religiose.

CREMASCHI Lisa (éd.), 2003, Regole monastiche femminili. Introduction d'Enzo Bianchi, Turin, Einaudi.

CREYTENS Raimondo, 1964, « La giurisprudenza della Sacra Congregazione del Concilio nella questione della clausura delle monache (1564-1576) ", in La Sacra Congregazione del Concilio. Quarto Centenario della Fondazione (1564-1964). Studi e ricerche, Cité du Vatican, p. 563-597.

DALL'OLIO Guido, 1995, « La disciplina dei religiosi all'epoca del Concilio di Trento : sondaggi bolognesi », Annali dell'istituto storico italo-germanico in Trento, XXI, p. 93-140.

DEL RE Niccolò, 1970 (3e édition mise à jour), La Curia Romana. Lineamenti storico-giuridici, Rome, Edizioni di Storia e Letteratura.

EVANGELISTI Silvia, 2004, « Monastic Poverty and Material Culture in Early Modern Italian Convents », The Historical Journal, 47, 1, p. 1-20.

-, 2007, Nuns. A History of Convent Life 1450-1700, Oxford et New York, Oxford University Press.

FRAGNITO Gigliola, 2003, La Bibbia al rogo : la censura ecclesiastica e i volgarizzamenti della Scrittura, 1471-1605, Bologne, Il Mulino.

GRECO Gaetano, 2006, La Chiesa in Occidente : istituzioni e uomini dal Medioevo all'Età moderna, Rome, Carrocci.

HILLS Helen, 2004a, « The Veiled Body : Within the Folds of Early Modern Neapolitan Convent Architecture », Oxford Art Journal, 27, 3, p. 269-290.

-, 2004b, Invisible City. The Architecture of Devotion in Seventeenth-Century Neapolitan Convents, Oxford et New York, Oxford University Press.

- (dir.), 2006, Architecture and the Politics of Gender in Early Modern Europe, Aldershot, Hampshire, Ashgate.

JACOBSON SCHUTTE Anne, 2006, «Legal Remedies for Forced Monachization in Early Modern Italy ", in Ronald K. Delph, Michelle M. Fontaine, John Jeffries Martin (dir.), Heresy, Culture, and Religion in Early Modern Italy. Contexts and Contestations, Kirksville, Missouri, Truman State University Press.

LAVENIA Vincenzo, 1998, « I diavoli di Carpi e il Sant'Uffizio (1636-1639) », in Mario Rosa (dir.), Eretici esuli e indemoniati nell'età moderna, Florence, Olschki.

LEHFELDT Elisabeth A., 2005, Religious Women in Golden Age Spain. The Permeable Cloister, Aldershot, Hampshire, Ashgate. 
LOMBARDI Teodosio, 1975, I francescani a Ferrara. IV : I monasteri delle Clarisse : S. Guglielmo, Corpus Domini, S. Bernardino, S. Chiara, Bologna.

LOWE Kate J. P., 1998, « Secular brides and convent brides : wedding cerimonies in Italy during the Renaissance and Counter-Reformation ", in Trevor Dean and Kate J. P. Lowe (dir.), Marriage in Italy, 1300-1650, Cambridge et New York, Cambridge University Press.

MEDIOLI Francesca, 1990, L'«Inferno monacale » di Arcangela Tarabotti, Turin, Rosenberg \& Sellier.

-, 1994, « Monacazioni forzate : donne ribelli al proprio destino », Clio. Rivista trimestrale di storia, 30, p. 431-454.

-, 1997, « La clausura delle monache nell'amministrazione della Congregazione romana sopra i Regolari ", in Gabriella Zarri (dir.), Il monachesimo femminile in Italia dall'alto medioevo al secolo XVII. A confronto con l'oggi, Negarine di San Pietro in Cariano (Verona), Il Segno dei Gabrielli Editore, p. 249-282.

MONSON Craig A., 1995, Disembodied Voices. Music and Culture in an Early Modern Italian Convent, Berkeley - Los Angeles, University of California Press.

NOVI CHAVARRIA Elisa, 2001, Monache e gentildonne : un labile confine : poteri politici e identità religiose nei monasteri napoletani, secoli 16.-17., Milan, Franco Angeli.

Ordine d'admettere le monache novizie alla professione per decreto dell'Ill.mo e Rev.mo Mons. Alessandro Marzi Medici...dato di nuovo in stampa..., Florence, 1709.

POMATA Gianna et ZARRI Gabriella (dir.), 2005, I monasteri femminili come centri di cultura fra Rinascimento e Barocco, Rome, Edizioni di Storia e Letteratura.

RUSSELL Camilla, sous presse, « Convent Culture in Early Modern Italy : Laywomen and Religious Subversiveness in a Neapolitan Convent ", in Practices of Gender in Late Medieval and Early Modern Europe, ed. by Megan Cassidy-Welch and Peter Sherlock, Turnhout, Brepols (Late Medieval and Early Modern Studies vol. 11).

SALLMANN Jean-Michel, 1989, Les sorcières fiancées de Satan, Paris, Gallimard.

SCATTIGNO Anna, 2005, « Una comunità testimone. Il monastero di Santa Maria degli Angeli e la costruzione di un modello di professione religiosa », in Gianna Pomata et Gabriella Zarri (dir.), I monasteri femminili come centri di cultura fra Rinascimento e Barocco, Rome, Edizioni di Storia e Letteratura, p. 175-204.

STOCCHETTI Denise, année académique 2003-2004, Lettere al monastero fiorentino delle Murate 1471-1630. Relazioni civili ed ecclesiastiche, Università degli Studi di Firenze, thèse de « laurea » sous la dir. de Gabriella Zarri.

-, 2005, «La fondazione del monastero fiorentino delle Murate e la pellegrina Eugenia », Archivio italiano per la storia della pietà, XVIII, p. 177-247.

TAMBURINI Filippo, 1995, Santi e peccatori : Confessioni e suppliche dai Registri della Penitenzieria dell'Archivio Segreto Vaticano (1451-1586), Milan, Istituto di Propaganda libraria.

WEAVER Elissa B., 2002, Convent theatre in early modern Italy : spiritual fun and learning for women, Cambridge et New York, Cambridge University Press.

ZARRI Gabriella, 1998, «Gender, Religious Institutions and Social Discipline : The Reform of the Regulars ", in Judith C. Brown \& Robert C. Davis (dir.), Gender and Society in Renaissance Italy, Londres et New York, Longman, p. 193-212.

-, 2000, Recinti. Donne, clausura e matrimonio nella prima età moderna, Bologne, Il Mulino. 


\section{NOTES}

1.COeD, sess. XXV, De regularibus. cap. V : 777-778.

2.Dall'Olio 1995.

3.Cette commission de cardinaux instituée par Pie IV en 1564 fut chargée de mettre fin aux questions concernant l'interprétation des décrets du Concile. Il s'ajouta à cette congrégation en 1586 une Congrégation des Évêques et Réguliers qui devait résoudre les questions ayant trait à la réforme du clergé régulier et aux rapports avec les évêques locaux ; par la suite, cette congrégation fut divisée en deux congrégations autonomes : l'une des Évêques, et l'autre des Réguliers. Cf. Del Re 1970 : 14-25 et Medioli, 1997.

4.Medioli 1990.

5.Medioli 1994.

6.La Sacra Penitenziaria, ministère de la Curie romaine, est le tribunal suprême de l'Église catholique pour le « for intérieur ». Ses compétences portent sur les absolutions des péchés, les censures et peines réservées au Saint-Siège, les dispenses

d'empêchements de mariages secrets et l'annulation des vœux; l'usage et la concession des indulgences. Cf. Del Re 1970 : 261-274. Sur les dispenses des vœux religieux aux XVe-XVIe siècles, Tamburini 1995.

7.Jacobson Schutte 2006.

8.Pour les grandes lignes, cf. Greco 2006.

9.Lehfeldt 2005.

10.Le concept de négociation est utilisé comme un a priori dans certaines des contributions du volume dirigé par Hills 2006.

11.Russell, sous presse.

12.Hills 2004a et 2004b.

13.Novi Chavarria 2001.

14.Evangelisti 2006, représente une bonne synthèse des études récentes sur les monastères de femmes.

15.Zarri 1998.

16.AEM, éd. Ratti, II.

17.La « ruota » ou « tour » est un dispositif de bois tournant, de la taille d'une petite fenêtre, situé dans l'atrium des monastères ; on y pose de petits objets que les religieuses peuvent saisir de l'intérieur sans être vues.

18. Ce sont des religieuses âgées qui doivent rester dans les parloirs afin d'écouter les conversations des professes avec les visiteurs autorisés.

19.Archivio Arcivescovile di Ferrara, Fondo documenti episcopali. Moniales. Licenze 1590, vol. 1.

20.Monastère florentin sur la fondation duquel cf. Stocchetti 2006.

21.Archivio di Stato di Firenze (ASF), Corporazioni Religiose Soppresse dal Governo francese (CRS). Murate n. 81, filza 100, fo 80 . Stocchetti a.a. 2002-2003.

22.Ainsi d'une veuve hébergée aux Murate en 1576 : ASF, CRS Murate n. 81, filza 100, f 153.

23.ASF, CRS Murate n. 81, filza 100, fo 161.

24.Biblioteca Apostolica Vaticana (BAV), Vaticano Latino 10444, Trattato sopra il governo de' Monasteri, fo 335.

25.Zarri 2000 : 173-176.

26.L'abbesse devait être « istruita ed esperta delle cose di Dio »; Cremaschi 2003 : 267. 
27.Cremaschi $2003: 268-269$.

28. Archivio Generale Arcivescovile di Bologna (AGAB), Miscellanee vecchie (MV) 914, 2, fo 3.

29. $A G A B, M V 914,2, f^{\circ} 6$.

30.AGAB, MV 914, Corrispondenze del Card. Ludovisi e Vicari Generali di Bologna (1621-1625), Ludovisi au vicaire, 9 décembre 1623, et le Memoriale joint, en date du $1^{\text {er }}$ décembre 1623.

31.La prétendante au siège abbatial aurait distribué de l'argent aux « sœurs jeunettes »; AGAB, MV 914, Francesco Ingoli au vicaire, 10 janvier 1624, et le Memoriale joint.

32.Arcangeli 1996.

33.Monson 1995.

34.Les élèves « dorment avec les nonnes, [et] certaines ont passé l'âge de vingt-cinq ans à l'encontre des ordonnances de la Sacrée Congrégation des Réguliers »; AGAB, MV 914, 7 mai 1625.

35.Barco $1609: 77$

36. AGAB, MV 914, 2, fo 6v.

37.AGAB, MV 914, 20 février 1627.

38.BAV, Vaticano Latino 10444, Trattato sopra il governo de' Monasteri, fo $337 \mathrm{v}$.

39.Weaver 2002.

40.Fragnito 2003.

41.BAV, Vaticano Latino 10444, Trattato sopra il governo de' Monasteri, fo338v.

42.Lombardi 1975 : 189-190.

43.Zarri 1998 et 2000 .

44.Cremaschi $2003: 210-211$.

45.Le monastère florentin des Murate, par exemple, était issu d'une première communauté de recluses ; Stocchetti 2005.

46.Ordine d'admettere le monache novizie alla professione... 1709.

47.Lowe 1998.

48.ASF, CRS San Pier Maggiore n. 55, $\mathrm{f}^{\circ} 242 \mathrm{r}^{\circ}$.

49.Scattigno 2005.

50.Pomata et Zarri 2005.

51.A Bologne, les extravagances de sœur Vittoria Felice Malvezzi mettaient en danger de mort ses consœurs ; AGAB, MV 914, 8 octobre 1565.

52.ASF, CRS San Pier Maggiore n. 55, fo $240 \mathrm{r}^{\circ}$.

53.BAV, Vaticano Latino 10444, Trattato sopra il governo de' Monasteri, $\mathrm{f}^{\circ} 346 \mathrm{v}^{\circ}$.

54."... perché spesse volte avviene che patischino le monache varii affetti d'utero che le fanno parere spiritate e veramente non sono »; AGAB, MV 914, 13 mai 1623.

55.Biondi 1991.

56.Sallmann 1989 ; Lavenia 1998.

57.AGAB, MV 914, 28 août 1626. 


\section{RÉSUMÉS}

La clôture des religieuses et les rapports de genre dans les couvents italiens (fin XVIe - début XVIIe siècle). L'article examine les changements dans les rapports de genre introduits par les normes du Concile de Trente sur les religieux, en particulier celles concernant la clôture des moniales. À Florence et Bologne prises comme terrains d'observation, les correspondances de l'archevêque Alessandro de'Medici avec le monastère florentin des Murate (1579-1606) et de l'archevêque de Bologne Ludovico Ludovisi avec ses vicaires (1624-1627) permettent de discuter la "perméabilité" de la clôture, un concept avancé par la critique récente. Les règles de la clôture n'ont interrompu ni les rapports étroits des monastères avec la cité et la société, ni la présence dans les couvents de filles à éduquer, veuves et femmes en rupture de ban conjugal, mais elles ont fortement réduit l'autonomie des religieuses, en déléguant aux évêques et à la Congrégation romaine sur les Religieux le contrôle de l'entrée dans les monastères. Les effets de la clôture tridentine à l'intérieur des monastères se traduisent par des changements significatifs dans la hiérarchie des offices, l'organisation de la communauté par classes d'âge et l'orientation des affects vers des personnes du même sexe. En ce qui concerne les rapports de genre, enfin, le rôle des confesseurs, référents masculins privilégiés des nonnes (que ce soit sur le plan spirituel ou sur celui des équilibres internes du monastère), se renforce.

The enclosure of nuns and gender relations in Italian convents (end of XVIth-beginning of XVIIth centuries). This article examines the shift in gender relationships introduced by the Council of Trent's regulations on religious orders, and on the enclosure of nuns in particular. Using the correspondence of Archbishop Alessandro de' Medici to the Florence convent of the Murate (1579-1606) and that of the Archbishop of Bologna Ludovico Ludovisi to his vicars (1624-1627), the author draws on material concerning two important Italian cities, Florence and Bologna, to discuss the concept of the "permeability" of enclosure recently developed by other historians. The article argues that the rules on enclosure did not entail a break in the strong relationship that convents had with the city and society, but rather drastically curtailed the independence of nuns by placing control of who entered the convent in the hands of bishops and the Roman Congregation on Religious. It then goes on to examine the effects of the Trent regulations on seclusion within convents noting a significant change in the hierarchy of the offices, the organization of the communities by age group and the affective orientation of religious persons toward the same sex. Lastly, with respect to gender relationships, the author stresses the strengthening of the role of the confessor as the privileged male referent of nuns in terms both of spirituality and of the internal equilibrium of the convent.

\section{INDEX}

Index géographique : Bologne, Florence

Keywords : Bologna, control of vocations, Post-Tridentine Church, religious hierarchy

Mots-clés : contrôle des vocations, Eglise post-tridentine, hiérarchie religieuse 


\section{AUTEUR}

\section{GABRIELLA ZARR}

Gabriella ZARRI est professeur d'histoire moderne à l'Université de Florence. Ses recherches portent sur les institutions ecclésiastiques et la vie religieuse entre XVe et XVIIe siècle, en particulier sur les ordres religieux et l'histoire des femmes. Parmi ses travaux : Le sante vive. Cultura e religiosità femminile nella prima età moderna (Turin, Rosenberg \& Sellier, 1990) ; Recinti. Donne clausura matrimonio nella prima età moderna (Bologne, Il Mulino 2000) ; La religione di Lucrezia Borgia. Le lettere inedite del confessore (Rome, Roma nel Rinascimento, 2006). 